\title{
Estimation par approche inverse d'un champ de transmissivité sur l'ensemble de l'aquifère du Continental Terminal (CT) d'Abidjan
}

\author{
Kouamé Auguste Kouassi ${ }^{1}$, William Francis Kouassi ${ }^{1}$, Oi Mangoua Jules Mangoua ${ }^{2}$, Philippe Ackerer ${ }^{3}$, \\ Gountôh Aristide Douagui ${ }^{1}$, and Issiaka Savané ${ }^{1}$ \\ ${ }^{1}$ UFR Sciences et Gestion de l'Environnement, Laboratoire Geosciences et Environnement, \\ Université NANGUI ABROGOUA, 02 BP 801 Abidjan 02, Côte d'Ivoire \\ ${ }^{2}$ UFR Environnement, Laboratoire des Sciences et Technologies de l'Environnement, \\ Université Jean Lorougnon Guedé Daloa, BP 150 Daloa, Côte d'Ivoire \\ ${ }^{3}$ Département «Laboratoire d'Hydrologie et de Géochimie de Strasbourg », \\ Université de Strasbourg, Strasbourg, France
}

Correspondence: Kouamé Auguste Kouassi (kouameauguste@yahoo.fr)

Published: 16 November 2021

Résumé. La caractérisation hydraulique des systèmes aquifères est importante pour le développement de scénarios d'exploitation et de stratégies de gestion des eaux souterraines. En particulier dans les aquifères lithologiquement hétérogènes, il ne faut pas négliger les variations de transmissivité $(T)$ à l'échelle locale. Les valeurs de transmissivité à l'échelle locale sont habituellement dérivées d'essais de pompage, mais dans la plupart des cas, leur nombre et leur disponibilité sont plutôt limités. De même, la mesure directe de la transmissivité sur l'ensemble d'un aquifère s'avère onéreux et quasi-impossible techniquement. Dans de telles situations, la modélisation hydrodynamique par approche inverse se présente comme la solution appropriée. Dans cet article, le champ de transmissivité réel de l'aquifère du Continental Terminal (CT) d'Abidjan est recherché par une paramétrisation multi-échelle qui permet de contourner le problème de changement d'échelle et de déterminer ce paramètre hydrodynamique sur l'ensemble de la nappe. Cette modélisation hydrogéologique a été réalisée à partir des modèles conceptuels Est-Ouest et Nord-Sud qui montrent que le CT est un aquifère constitué de deux couches principales $\mathrm{n} 3$ et $\mathrm{n} 4$, respectivement formées de sables grossiers de $90 \mathrm{~m}$ d'épaisseur et de sables fins à moyen de $80 \mathrm{~m}$ d'épaisseur. Le calage du modèle bicouche s'est effectué en régime transitoire de 12 pas de temps mensuels avec des données piézomètriques de 24 piézomètres, des données de production de 71 forages et des valeurs de transmissivité connues de 40 forages dans un maillage élément fini constitué de 534 mailles et 320 noeuds. Ainsi, ce modèle de la nappe du CT a identifié une structure de dimension 153 nœuds comme la structure la plus proche de celle de l'aquifère du CT. Les valeurs de $T$ variant entre $5,4.10^{-5}$ et $1 \mathrm{~m}^{2} \mathrm{~s}^{-1} \mathrm{du}$ champ de transmissivité associé à cette dimension optimale, ont été comparées aux valeurs de $T$ d'autres champs de transmissivités publiées dans des études en Afrique et dans le monde. Ces valeurs identifiées sont plausibles et présentes une bonne structure dans l'ensemble. 


\section{Introduction}

Les nombreuses sollicitations de l'eau ont pour conséquence de diminuer la réserve d'eau disponible partout dans le monde (De Lacour, 2012). Abidjan, la plus grande ville de Côte d'Ivoire n'échappe pas à cette réalité mondiale. Elle représente moins de $1 \%$ du territoire national mais produit et consomme à elle seule près de $70 \%$ de l'eau potable du pays (DHH, 2001; SODECI, 2017). Abidjan est essentiellement alimentée en eau potable à partir des eaux souterraines de la nappe du Continental Terminal (CT). Les fortes augmentations de la demande en eau d'Abidjan (SOGREAH, 1997; Kouassi, 2013; SODECI, 2017) avec très peu de recharge ont mis à rude épreuve ses eaux souterraines, ce qui s'est traduit par une baisse générale des niveaux statiques (Ahoussi, 2008; Kouassi, 2013). Face à la pression démographique que connait Abidjan et au besoin en eau croissant de sa population nous nous sommes interrogés sur la capacité de la nappe d'Abidjan à produire suffisamment d'eau pour satisfaire ces multiples besoins. Cette question trouve sa réponse dans la connaissance de la transmissivité $(T)$ sur l'ensemble de l'aquifère du Continental Terminal. En effet, $T$ est le principal paramètre hydraulique qui contrôle les prélèvements des eaux souterraines. La méthode la plus fiable pour estimer la transmissivité d'un aquifère est d'effectuer une analyse d'essai de pompage ponctuel (Mjemah et al., 2009). Toutefois, cette approche de mesure directe de la transmissivité sur l'ensemble de la nappe nécessite des ressources techniques et financières énormes qu'il est pratiquement et économiquement impossible de satisfaire (Kouassi, 1999). Dans de tels cas, la modélisation hydrodynamique via une approche inverse qui consiste à rechercher les valeurs inconnues des propriétés de l'aquifère (Transmissivité $T$ ) en utilisant les variables d'état de celui-ci (charge hydraulique h) connues aux points de mesure, s'impose comme la solution idoine. L'objectif de ce travail est d'estimer un champ de transmissivité sur l'ensemble de l'aquifère du Continental Terminal d'Abidjan. Ce champ renseignera donc les gestionnaires de la nappe d'Abidjan sur la disponibilité et les potentialités de captage de la ressource en eau dans cet aquifère. L'identification de ce champ a été possible grâce à un modèle numérique réalisée à partir du code HPP-GMS basé sur la méthode des éléments finis de Galerkin développée autour d'éléments triangulaires.

\section{Methodologies}

Dans cette étude, la détermination du champ de transmissivité existant de l'aquifère du Continental Terminal s'est construite autour de trois principales étapes que sont la caractérisation physique de l'aquifère (modèle conceptuel), la conceptualisation du modèle numérique de l'aquifère (maillage, données d'entrées, conditions aux limites etc. ...) et la recherche de la structure et de la dimension la plus proche du milieu naturel suite à une série de simula- tions des charges par la méthode inverse de paramétrisation multi-échelle.

\subsection{Caractérisation physique et modèle numérique de l'aquifère du CT}

La caractérisation physique de l'aquifère du CT s'est résumé par des modèles conceptuels servant à simplifier la structure réelle et complexe de l'aquifère en des schémas géologiques fonctionnels et par des hypothèses de fonctionnement établis. Ainsi, le modèle conceptuel de l'aquifère du CT réalisé à partir de 185 fiches techniques de forages, présente une structure bicouche dont l'épaisseur totale varie entre 30 et $160 \mathrm{~m}$ dans la direction Est-Ouest et varie également entre 30 et $120 \mathrm{~m}$ dans la direction Nord-Sud (Fig. 1).

Cet ensemble ne forme qu'un seul aquifère libre constitué majoritairement sables fins à moyens et de sable grossier avec une épaisseur moyenne captante de $90 \mathrm{~m}$. Les valeurs de transmissivités ponctuelles déduites des essais de pompages de 40 forages irregulièrement repartis, oscillent entre les gammes $10^{-6}$ et $10^{-1} \mathrm{~m}^{2} \mathrm{~s}^{-1}$. Le modèle numérique de la nappe du CT d'Abidjan est en maillage éléments finis comportant 534 mailles pour 320 nœuds (Fig. 1). Les conditions aux limites se résument à des potentiels imposés aux frontières Nord et Sud, à des flux imposés nuls aux limites Ouest et Nord-Est dictés par la perpendicularité des lignes de courants aux equipotentielles et à des flux imposés non nuls entrant à la frontière Sud-Est. La prise en compte des échanges entre la nappe du Continental Terminal et ses rivières ne concerne que les rivières Gbangbo, Banco et Bété. Ces échanges mettent à contribution le niveau de la nappe, la cote du fond de la rivière et la cote de sa surface libre, et un coefficient d'échange entre les deux milieux.

\subsection{Estimation de la transmissivité}

En considérant l'équation de diffusivité, l'estimation des transmissivités se résument à la minimisation de la fonction objectif sur les charges et sur les transmissivités. La fonction objectif, sert de critère pour déterminer la meilleure solution à un problème d'optimisation. Concrètement, elle indique le degré de rapprochement entre les simulations du modèle et les observations de l'état du système. Pour un domaine de calcul de 534 mailles, seulement 40 mailles se sont vues attribuées une valeur de transmissivité ponctuelle issu d'une analyse d'essai de pompage. Pour réaliser le modèle hydrodynamique, les données piézométriques sont fournies par les mesures effectuées sur 24 piézomètres reparties sur la nappe du CT sur une période de 1 an réparties en 12 pas de temps mensuel. Les sollicitations de la nappe se résument essentiellement à la production journalière des 71 forages fonctionnels des neuf (9) champs captants SODECI d'Abidjan à l'exception des forages du champ captant du plateau qui est fermé. 

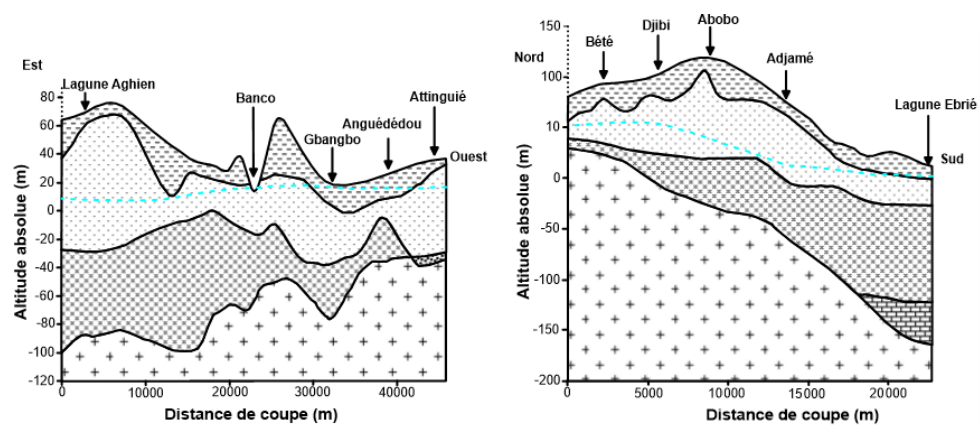

Niveau piézométrique Sable argileux

Schistes altérés sableux 覽 Calcaires, sables, argiles

Socle cristallin et cristallophylien

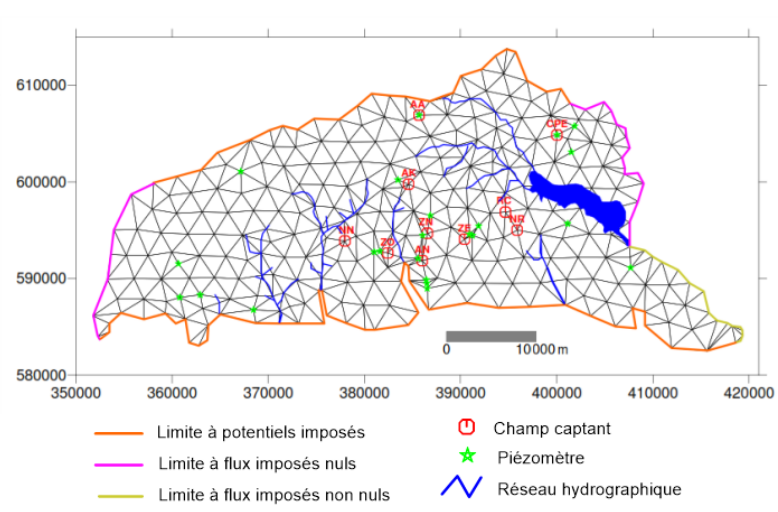

Figure 1. Schéma conceptuel hydrogéologique Est-Ouest et Nord-Sud et Conceptualisation du modèle numérique de l'aquifère du Continental Terminal d'Abidjan.

\subsubsection{Paramétrisation multi-échelle}

La détermination du champ de transmissivités réel nécessite, la recherche de la structure et de la dimension la plus proche du milieu naturel. Afin d'atteindre cet objectif, nous adoptons la méthode de paramétrisation multi-échelle non automatique dont l'idée est d'utiliser dès le départ plusieurs structures différentes pour générer un ensemble de solutions possibles. Cette approche consiste à représenter la structure du milieu par des structures de plus en plus élaborées, au cours de l'identification des paramètres du modèle. Cette démarche se traduit par une augmentation de la dimension du modèle de paramétrisation, par affinement du maillage, d'une étape à la suivante (Kouassi, 2010). C'est donc dans cet état d'esprit que l'identification des transmissivités du modèle de la nappe d'Abidjan s'est effectuée autour de huit structures de base dénommées structure 100, structure 200 jusqu'à structure 800. La proposition de ces structures de départ a été guidée par l'exploitation simultanée de plusieurs informations sur le milieu physique (transmissivités ponctuelles, débits spécifiques, données géologiques, occupation du sol etc. ...), sans oublier la connaissance générale que nous avons du secteur à partir des études antérieures (Loroux, 1978; SOGREAH, 1996; Kouassi et al., 2010; Kouassi, 2013). Ainsi à l'aide de ces informations nous avons raffiné ou non certaines zones du domaine d'étude pour générer les différentes structures. Chaque structure est par la suite constituée d'une série de maillage de paramétrisation de dimension graduellement croissante à tester. L'ensemble des maillages de paramétrisation à tester des huit structures confondus est de 115 maillages.

La procédure d'identification multi-échelle non automatique démarre par un maillage de grande dimension, composé de quatre mailles et de cinq nœuds couvrant tout le domaine de calcul (Ackerer et al., 1996). Après cette première étape, nous enchaînons directement avec la série de maillages des huits structures de base prédéfinis grâce à l'état de connaissance de l'aquifère (Fig. 2).

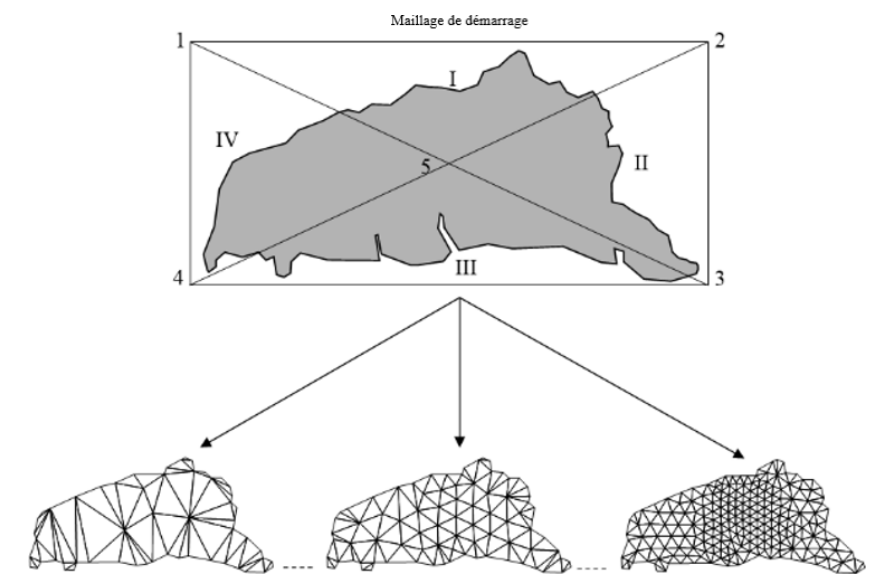

Figure 2. Procédure de la paramétrisation multi-échelle non automatique.

\subsubsection{Calage et validation du modèle}

Le calage du modèle vise à reproduire au mieux le fonctionnement hydrodynamique de la nappe en ajustant les différents paramètres que l'on estime pouvoir faire varier tout en restant dans la gamme des valeurs réalistes (Leduc, 2005). Pour améliorer les résultats de la simulation, en plus de la transmissivité, les coefficients d'échange nappe-rivières, les flux, la surface libres des rivières et la porosité ont été ajustés.

Une fois le calage lancé, on observe qu'au fur et à mesure que le nombre de paramètres augmente, la fonction objectif diminue. Elle diminue très rapidement au début et se stabilise autour d'une valeur au-delà d'une certaine dimension de la paramétrisation (nombre de paramètres). Tous les maillages de paramétrisation qui ont donné ces résultats satisfaisants sur la valeur de la fonction objectif peuvent être retenus comme approximations possibles de la structure du milieu étudié. Dans ce cas, le seul critère retenu est la valeur de la fonction objectif. On aurait alors une multitude de solutions pour un problème qui en réalité n'en a qu'une 
seule puisque la structure réelle est unique. Pour limiter les effets de l'inévitable non-unicité de la solution du problème inverse, il est donc indispensable de trouver un critère autre que la fonction objectif. Ce critère servira à évaluer la qualité du jeu de paramètres associé à chaque modèle de paramétrisation et à réduire le nombre de solution au problème inverse. Pour apprécier la qualité de chaque maillage de paramétrisation, nous déterminons donc deux critères de performance.

Le premier critère est celui classique sur les charges, qui indique dans quelle mesure le modèle a pu simuler l'état du système sur la base des données disponibles. Ce critère désignant erreur de modèle est noté Erreur_H (Eq. 1).

Erreur_H $=\sqrt{J(P) / N \cdot N_{\mathrm{p}}}$

Avec :

$J(P)=\sum_{n=0}^{N-1}\left\|H_{\mathrm{c}}^{n+1}-H_{\mathrm{m}}^{n+1}\right\|^{2}$

$H_{\mathrm{c}}$ : Vecteur des charges simulées par le modèle aux points d'observations, $H_{\mathrm{m}}$ : Vecteur des charges mesurées sur le terrain aux points d'observations, $N$ : Nombre de pas de temps, $N_{\mathrm{p}}$ : Nombre de points d'observation de la charge sur le terrain.

Erreur_H représente la moyenne sur tous les points de mesure et à tous les pas de temps, de l'écart entre les charges simulées et les charges mesurées.

Le deuxième critère porte sur la qualité des paramètres estimés. Ainsi, le critère Erreur_T qui représente la moyenne des écarts relatifs quadratiques entre les transmissivités identifiées et les transmissivités connues, prend la forme de l'équation (2) suivante :

Erreur_T $=100 \cdot \sqrt{\frac{1}{N_{\mathrm{e}}} \sum_{j=1}^{N_{\mathrm{e}}}\left(\frac{T c_{j}-T m_{j}}{T m_{j}}\right)^{2}}$

Avec : $T c_{j}$ : Transmissivité identifiée sur l'élément $j$ du maillage de calcul; $\mathrm{Tm}_{j}$ : Transmissivité du champ connu pour l'élément $j, N_{\mathrm{e}}$ : Nombre total d'éléments du maillage de calcul.

Dès lors que les calages successifs sont réalisés, nous disposons pour chaque maillage de paramétrisation d'une valeur de Erreur_H et d'une valeur de Erreur_T. Ces deux critères Erreur_H et Erreur_T sont alors représentés dans un même graphe en fonction de la dimension de la paramétrisation. La représentation de Erreur_H et de Erreur_T permet dans la mesure du possible, de sélectionner la structure et la dimension optimale du modèle de paramétrisation qui convient pour le calage du modèle (Kouassi, 1999). A l'analyse des courbes de Erreur_H et Erreur_T, nous couplerons les informations recueillies aux résultats de la comparaison entre les charges simulées et les charges observées. Pour encore réduire le nombre de solutions de l'identification des transmissivités et également servir de facteur de validation

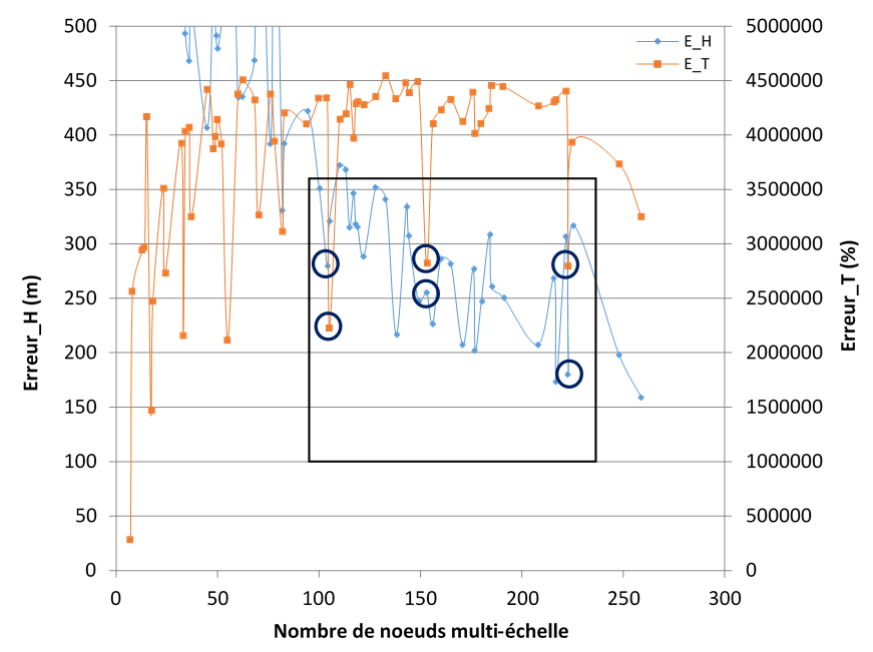

Figure 3. Représentation locale de la fraction de Erreur_H stable et de Erreur_T en fonction du nombre de paramètres multi-échelle.

du champ de transmissivité identifié, des valeurs de transmissivité n'ayant pas servi dans la mise en place du modèle vont être positionnées sur les probables champs de transmissivité solutions du problème inverse. L'objectif de superposer les transmissivités connues sur les champ transmissivités calculées est de rechercher le champ de transmissivité identifié qui reproduit le mieux les valeurs réelles des transmissivités de la nappe d'Abidjan.

\section{Resultats et discussions}

\subsection{Identification du champ de transmissivité}

L'analyse des valeurs de Erreur_H et de Erreur_T comprises entre 0 et $5.10^{6}$ issus des différents calages réalisés a permis de choisir trois maillages (maillages 105; 153 et 223 nœuds de la structure 700) de paramétrisation pour identifier les paramètres du modèle (Fig. 3). Les valeurs de transmissivités estimées oscillent entre $5,4 \cdot 10^{-5}$ et $1 \mathrm{~m}^{2} \mathrm{~s}^{-1}$. Parmi ces trois maillages présélectionnés (Fig. 4), nous ne pouvons pas d'ores et déjà choisir l'un d'entre eux pour représenter les paramètres du modèle car le minimum de E_H n'est pas distinguable de manière incontestée. Ainsi, il nous faut des informations qualitatives complémentaires.

\subsection{Détermination de la structure et de la dimension optimale du modèle}

L'analyse des courbes de Erreur_H et de Erreur_T en fonction du nombre de paramètres a permis de disposer dans un premier temps d'un ensemble de maillages solutions possibles du problème inverse et ensuite de réduire cet ensemble de solutions à trois maillages proches de la structure vraie. Il a été cependant impossible de déterminer une structure et une dimension unique proche de la structure réelle de la 

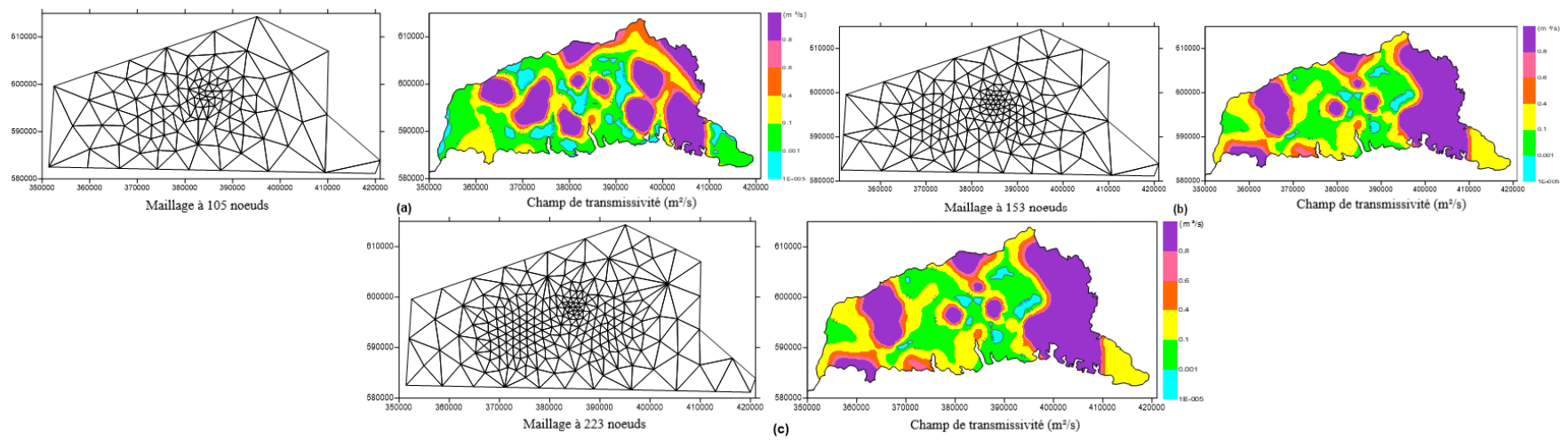

Figure 4. Maillages de paramétrisation et champs de transmissivités associés : (a) Maillage de 105 nœuds et son champ de transmissivité ; (b) Maillage de 153 nœuds et son champ de transmissivité; (c) Maillage de 223 nœuds et son champ de transmissivité.

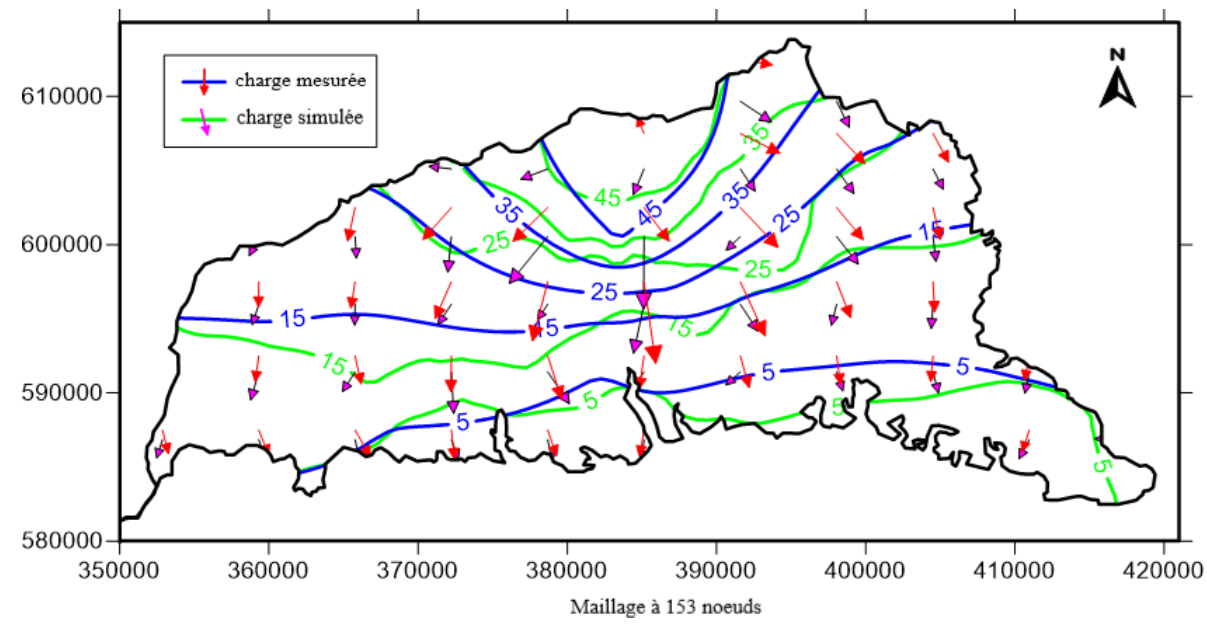

Figure 5. Comparaison des charges mesurées et simulées en juin 2005 dans le maillage de 153 nœuds

nappe parmi ces trois maillages. En regardant dans le détail la portion de la courbe E_H solution du problème inverse précédemment identifiés, il n'est toujours pas possible de départager concrètement les trois champs de transmissivités déterminés et de faire un choix unique. En l'absence d'autres critères de différenciation de ces maillages indiqués, la comparaison des charges simulées associées à chaque maillage de paramétrisation, aux charges mesurées aux points d'observation, est exploitée. Dans l'ensemble, les trois maillages de paramétrisation identifiés restituent quasi fidèlement le sens d'écoulement général de la nappe. Toutefois, il faut noter que les lignes piézométriques issues des charges calculées du maillage de 153 nœuds se rapprochent plus des lignes de références (Fig. 5). La comparaison des charges observées et des charges calculées a permis de déterminer la dimension de 153 nœuds de la structure 700 comme étant la dimension optimale du modèle de la nappe du Continental Terminal.

$\mathrm{Au}$ terme de nos investigations, nous proposons le champ de transmissivité associés au maillage de 153 nœuds comme la structure la plus proche de la structure réelle de l'aquifère du Continental Terminal d'Abidjan (Fig. 4b).

\section{Validation des transmissivités}

La validation du champs identifié montre que les transmissivités mesurées du champ captant d'Anguédédou appartenant aux gammes de $10^{-3}$ et $10^{-2} \mathrm{~m}^{2} \mathrm{~s}^{-1}$ sont situées dans la classe de transmissivité de $10^{-3}$ à $10^{-1} \mathrm{~m}^{2} \mathrm{~s}^{-1}$ du champ de transmissivité identifié calculés à l'issu des simulations (Fig. 6). A la fin de ce test de validation, nous pouvons donc déduire que le champ de transmissivité issu du maillage de 153 nœuds à la Fig. 7 représente au mieux la structure vraie de l'aquifère du Continental Terminal d'Abidjan.

\subsection{Discussion}

L'analyse des critères E_H et E_T à la suite des séries de calage a permis de déterminer les maillages à 105, 153 et 223 nœuds de la structure 700 comme solutions du problème inverse. Comme cela est très souvent le cas dans de nom- 


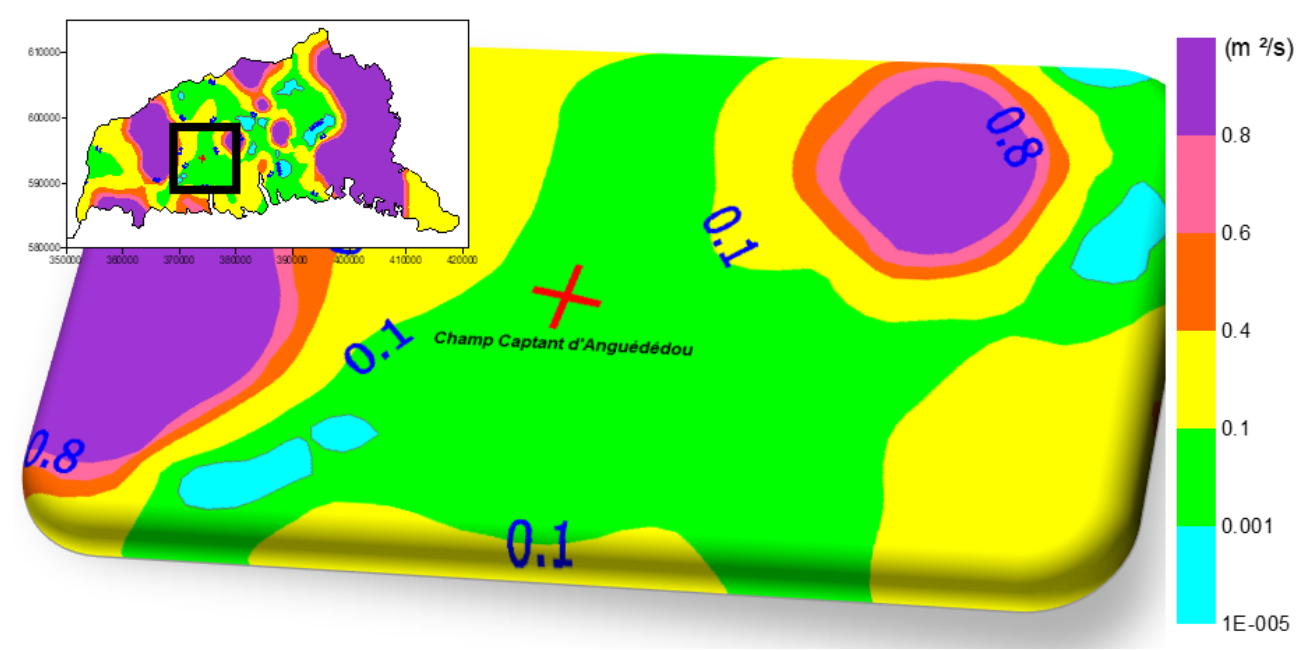

Figure 6. Positionnement du champ captant d'Anguédédou sur le champ de transmissivité identifié.

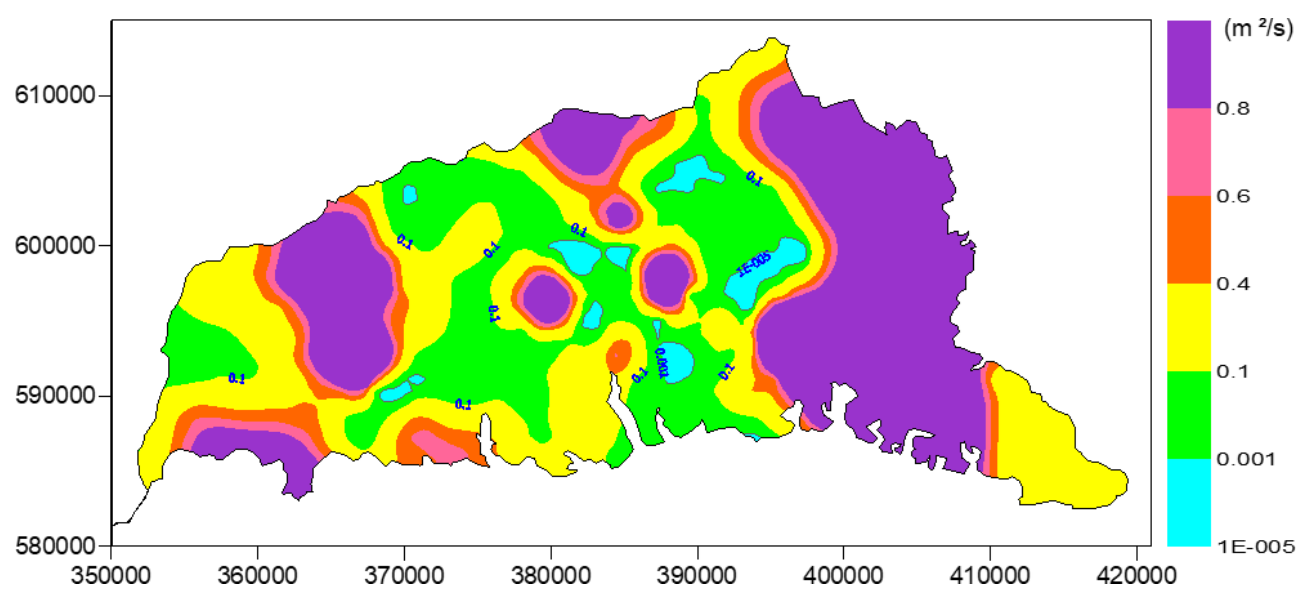

Figure 7. Champ de transmissivité identifié comme la structure la plus proche de la structure de l'aquifère du Continental Terminal d'Abidjan.

breux domaines de géosciences, nous sommes typiquement en présence d'un problème inverse mal posé car il n'admet pas de solution unique et stable. Alors, le seul moyen de réduire ces incertitudes serait d'ajouter de l'information soit sous forme de piézométries observées, soit sous forme d'essais de pompages. Les valeurs des fonctions objectifs E_H et E_T comprises entre 0 et $5.10^{6}$ sont restées élevées comme dans les travaux de Lehmann (1996) où la fonction objectif $O(p)$ est parfois allée jusqu'à $10^{9}$. Les raisons des fonctions objectif élevées, sont probablement liées à la complexité du milieu d'étude naturel et au nombre insuffisant des points de mesures réelles. Les champs de transmissivités calculées, associés aux trois maillages sélectionnés présentent une bonne structure dans l'ensemble et cela en conformité avec l'hydrodynamique de la nappe du CT. Ces transmissivités estimées $\left(5,4 \cdot 10^{-5} \leq T<1 \mathrm{~m}^{2} \mathrm{~s}^{-1}\right)$ s'inscrivent en partie dans la gamme des transmissivités mesurées dans plusieurs aquifères sédimentaires continentaux ou côtiers de l'Afrique et de celle mesurée par d'autres auteurs (Loroux, 1978; Scet Ivoire et al., 1980) sur le bassin sédimentaire côtier ivoirien. Ainsi, les valeurs de transmissivités estimées sur la majeure partie de l'aquifère du CT d'Abidjan variant entre $10^{-1}$ et $1 \mathrm{~m}^{2} \mathrm{~s}^{-1}$ sont possibles. Cette démonstration permet également de montrer que l'aquifère du CT est puissant et très productif par endroits. Les séries de calage ont permis de choisir le maillage de 153 nœuds comme la dimension optimale du modèle. Le champ de transmissivités associés à cette dimension optimale est défini comme la structure la plus proche de la structure vraie de l'aquifère du CT. En conséquence, le champ de transmissivité identifié pourra servir de guide dans l'implantation des forages à gros débits sur la nappe d'Abidjan et en retour le taux de réussite de ces implantations permettra d'évaluer le niveau de crédibilité du champ identifié. 


\section{Conclusions}

La modélisation de l'écoulement de la nappe du Continental Terminal a permis de mettre en place un modèle numérique d'écoulement constitué d'un maillage de 534 mailles et de 320 nœuds. Par ailleurs, l'identification des paramètres autour de huit structures de départ s'est soldée par le choix de la structure 700 de dimension 153 nœuds comme la structure la plus proche de la structure vraie. Le champ de transmissivité associé à ce maillage présente de bonnes valeurs d'ensemble variant entre $5,4 \cdot 10^{-5} \leq T<1 \mathrm{~m}^{2} \mathrm{~s}^{-1}$ dont l'essentiel des valeurs oscillent entre $10^{-3}$ et $1 \mathrm{~m}^{2} \mathrm{~s}^{-1}$. Ce champ de transmissivité estimé sur l'aquifère du Continental Terminal d'Abidjan renseigne sur les énormes potentialités d'implantation des forages et le captage de la ressource en eau sur l'ensemble de cet aquifère.

Disponibilité du code. Le code de modelisation dont les réferences suivent : HPP-GMS plus Hydrology Pre/Post Processor V 4.50 @ Copyright 1996, 1997 MSB All rights reserved. nous été offert gracieusement par le Directeur de Recherche CNRS Philippe Ackerer du Laboratoire d'hydrologie et de géochimie de Strasbourg (UMR 7517).

Disponibilité des données. En plus des données recoltées sur le terrain, les autres données utilisées ces travaux sont accessibles au public et ont été collectées auprès des structures étatiques et privés ci-dessous : la Direction de l'Hydraulique Humaine (DHH), le Centre de cartographie et de télédetection (CCT), l'entreprise Forage Rationnel Construction (FORACO), la Société de Distribution d'Eau de Côte d'Ivoire (SODECI), et la Société de développement Minier (SODEMI).

Collaborateurs. KAK : Organisation et exécution des travaux de terrain, collecte de données, caractérisation de l'aquifère par un modèle conceptuel, conceptualisation du modèle numérique et rédaction de l'article. WFK : Appui à la réalisation du modèle conceptuel et du modèle numérique de l'aquifère et correction de l'article. NB : Co-auteur décédé le mardi 19 octobre 2021 Oi Mangoua Jules Mangoua : Participation aux travaux de terrain, appui à la réalisation du modèle conceptuel et correction de l'article. PA : Mise à disposition du code HPP-GMS plus V 4.50, appui à l'usage du code et à la réalisation du modèle numérique de l'aquifère. GAD : Participation aux travaux de terrain et correction de l'article. IS : Orientation et correction de l'article.

Intérêts concurrents. Les auteurs déclarent qu'ils n'ont aucun conflit d'intérêts.

Clause de non-responsabilité. Publisher's note : Copernicus Publications remains neutral with regard to jurisdictional claims in published maps and institutional affiliations.
Déclaration du numéro spécial. This article is part of the special issue "Hydrology of Large River Basins of Africa". It is a result of the 4th International Conference on the "Hydrology of the Great Rivers of Africa", Cotonou, Benin, 13-20 November 2021.

Remerciements. Mes remerciements vont l'endroit du Laboratoire Géosciences et Environnement de l'Université NANGUI ABROGOUA (Côte d'Ivoire), du Laboratoire d'Hydrologie et géochimie de Strasbourg (France) et des structures telles que la DHH, le CCT, la SODECI et l'ONEP pour leur appui logistique dans la réalisation de ce travail. Au Dr William Francis KOUASSI, je tiens à dire particulièrement merci de m'avoir initié à la modélisation hydrogéologique, transmis l'abnégation et la hargne au travail. Fauché le 19 octobre 2021 dans la plénitude de ton art des sciences de l'eau, jamais je n'oublierai ton humanité et ta sollicitude pour ton prochain cher Maître. Repose en paix.

\section{Références}

Ackerer, P. H., Siegel, P., and Blaschke, A. P. : Inverse problem applied to groundwater flow and transport equations using a downscaling parameterization, Proc. of "Computational Methods in Water Resources XI", Cancun, Mexique, 22-26 July, edited by : Aldama, A., Aparicio, J., Brebbia, C., Gray, W. G., Herrera, I., and Pinder, G., Computational Mechanics Publ., 321-328, 1996.

Ahoussi, K. E. : Evaluation quantitative et qualitative des ressources en eau dans le Sud de la Côte d'Ivoire. Application de l'hydrochimie et des isotopes de l'environnement à l'étude des aquifères continus et discontinus de la région d'Abidjan-Agboville, Thèse unique de l'université de Cocody, Côte d'Ivoire, 283 pp., 2008.

De Lacour, G. : Les nappes phréatiques mondialement à la baisse, le journal de l'environnement, 26755, accessible à l'adresse suivante : http://www.journaldelenvironnement. net/article/les-nappes-phreatiques-mondialement-a-la-baisse, la date du dernier accès : 8 décembre 2012.

DHH (Direction de l'Hydraulique Humaine) : Hydraulique $\mathrm{Hu}-$ maine en Côte d'Ivoire, Ministère des Infrastructure Economiques, Abidjan, 66 pp., 2001.

Kouassi, K. A. : Modélisation hydrodynamique en milieu poreux saturé par approche inverse via une paramétrisation multiéchelle : Cas de l'aquifère du Continental Terminal d'Abidjan (Côte d'Ivoire), thèse unique en sciences et gestion de l'environnement de l'université Nangui Abrogoua, 266 pp., 2013.

Kouassi, W. F. : Estimation des paramètres hydrogéologique par approche inverse, Mise en œuvre d'une approche multi-échelle sur un secteur de la nappe d'Alsace, Thèse Université Louis Pasteur-Institut de Mécanique des Fluides, UMR 7507 UPLCNRS, Strasbourg, 165 pp., 1999.

Kouassi, K. A., Kouassi, F. W., Goula, B. T. A., Kouame, K. I., Dibi, B., and Savane, I. : Conceptual model of Ivorian sedimentary costal basin : case of Abidjan Continental Terminal aquifer, Eurp. J. Sci. Res., 44, 400-419, 2010.

Leduc, C. : Modélisation hydrogéologique Orsay 2004-2005, Notes de cours de modélisation numérique en hydrogéologie, 16 pp., 2005.

Lehmann, F. : Hydrodynamique en milieux poreux hétérogènes non saturés : identification des paramètres par approche inverse, 
Thèse Université Louis Pasteur - Institut de Mécanique des Fluides, URA CNRS 854, Strasbourg, 191 pp., 1996.

Loroux, B. F. E. : Contribution à l'étude hydrogéologique du bassin sédimentaire côtier de Côte d'Ivoire, Thèse de Doctorat de 3ème cycle, Université de Bordeaux I, France, 93 pp., 1978.

Mjemah, I. C., Van Camp, M., and Walraevens, K. : Groundwater exploitation and hydraulic parameter estimation for Quaternary aquifer in Dar-es-Salaam, Tanzania, J. Afr. Earth Sci., 55, 134146, 2009.

Scet Ivoire, Sodeci, Holfelder, Scet International : Alimentation en eau potable d'Abidjan. Plan directeur et étude de la nappe et sa protection contre la pollution. Etude hydrogéologique de la nappe d'Abidjan : ressource et exploitation optimale de la nappe. Rapports no. 2 et no. 3, République de Côte d'Ivoire, Ministère des travaux publics, des transports, de la construction et de l'urbanisme : Direction Centrale de l'Hydraulique, 44 pp., 1980.
SODECI : Rapport d'Activités 2017, Société de Distribution d'Eau de Côte d'Ivoire, 60 pp., 2017.

SOGREAH : Etude de la gestion et de la protection de la nappe assurant l'alimentation en eau potable d'Abidjan, Etude sur modèle mathématique, Rapport de phase 1 et 2, République de Côte d'Ivoire, Ministère des infrastructures Economiques, Direction et Contrôle des Grands Travaux (DGCTX), 22 pp., 1997. 